\title{
Development of marine observation system using LPWA communication system for marine IoT service
}

\author{
Se-Hoon Kim ${ }^{1}$, Min-Ho Jeon ${ }^{2}$, Yeon-Ju $\mathrm{Jo}^{3}$, Chang-Heon $\mathrm{Oh}^{4}$ \\ ${ }^{1}$ Corporate Technology Institute, Company of Technology and Information Emotion, Korea \\ ${ }^{2,3}$ Corporate Technology Institute, Company of Radio Communication Network, Korea \\ ${ }^{4}$ School of Electrical, Electronics and Communication Engineering, Korea University of Technology and Education, Korea
}

\begin{abstract}
Article Info
Article history:

Received Feb 10, 2020

Revised Mar 9, 2020

Accepted Apr 5, 2020

\section{Keywords:}

Location tracking

Low power wide area

Marine observation

Maritime IOT service

Ship resource
\end{abstract}

\section{Corresponding Author:}

Chang-Heon Oh,

School of Electrical, Electronics and Communication Engineering,

Korea University of Technology and Education,

1600, Chungjeol-ro, Byeongcheon-myeon, Dongnam-gu, Cheonan-si,

Chungcheongnam-do, 31253 Republic of Korea.

Email: choh@koreatech.ac.kr

\begin{abstract}
Because oceans account for $70.8 \%$ of the Earth's surface, various Internet-ofThings (IoT) applications for the marine industry are being researched and developed. The success of an IoT technology in the maritime industry depends on the location tracking technology used and how far it can communicate. In this study, we implemented a marine observation system using low power wide area (LPWA) communication technology to provide maritime IoT services. The proposed marine observation system consists of a marine IoT module and an ocean observation monitoring system. The information collected is GPS information and communication signal reception strength. As a result of an actual field test, we were able to obtain the distance to the gateway by measuring the position through the marine IoT module, and we found that the intensity of communication was proportional to the change in sea level.
\end{abstract}

Copyright $(2020$ Institute of Advanced Engineering and Science. All rights reserved.

\section{INTRODUCTION}

Recently, various Internet-of-Things (IoT) applications have emerged, and there has been a significant increase in the number of devices used in everyday life and industry [1-4]. Statista, a statisticsspecialized agency, estimates that by 2025, 75.4 billion IoT devices will be distributed worldwide [5]. Therefore, various studies are underway that are investigating IoT characteristics, including low power communication, platforms, big data, and the cloud [2, 6-9]. LPWA targets low-data speed, low-power, and low-through applications, and can be connected as far as tens of kilometers using a sub-GHz band, which is less congested than the traditional Industry, Science, Medical (ISM) band [10, 11]. Although various studies and developments have been made on LPWA based IoT application programs, research and development of devices for application to the marine environment have not been conducted yet [12-14].

In this study, we developed a marine observation system to apply LPWA-based IoT applications to a marine environment. We evaluated the performance of the developed system using the collected information. As a result of actual field experiments, we determined the position of the marine IoT module using GPS information. Further, it is shown that the distance between each other can be obtained by using the GPS data of the gateway and buoy. As a result of the comparison between the sea level change data and the reception intensity through the LPWA communication, it was found that the change in the sea level can be observed using the reception intensity of the communication. Using these results, it is possible to observe the ocean by collecting various information regarding nearby oceans. 
The rest of this paper is organized as follows. In Section 2, we describe a marine IoT module that collects information from the proposed system. Section 3 explains marine observation systems based on the maritime IoT module. In Section 4, we construct a test environment to evaluate the performance of the developed system and the performance of the developed system is evaluated. Finally, we provide the conclusions in the last section.

\section{DEVELOPMENT OF EQUIPMENT AND COMMUNICATION}

LPWA includes SigFox, LoRa, Weightless, and Ingenu using the 169, 433, 868/915 MHz, and 2.4 GHz unlicensed bands, respectively; and LTE-M, EC-GSM, and NB-IoT using licensed bands depending on the operating area. Major LPWA technologies and their characteristics are shown in please refer to the following paper. $[12,13-21]$.

Prior to developing the maritime observation system, we developed a marine-specific IoT module based on LPWA. This module, which is specialized for use in the ocean, can be understood using the circuit shown in Figure 1 and Figure 2. The power source is operated using a magnet to prevent leakage caused by the power source of the IoT device placed in the sea. Since it is used in the ocean for a long time, the MCU can control the power to the GPS, such that it can receive its position at the optimized position in the arranged place.

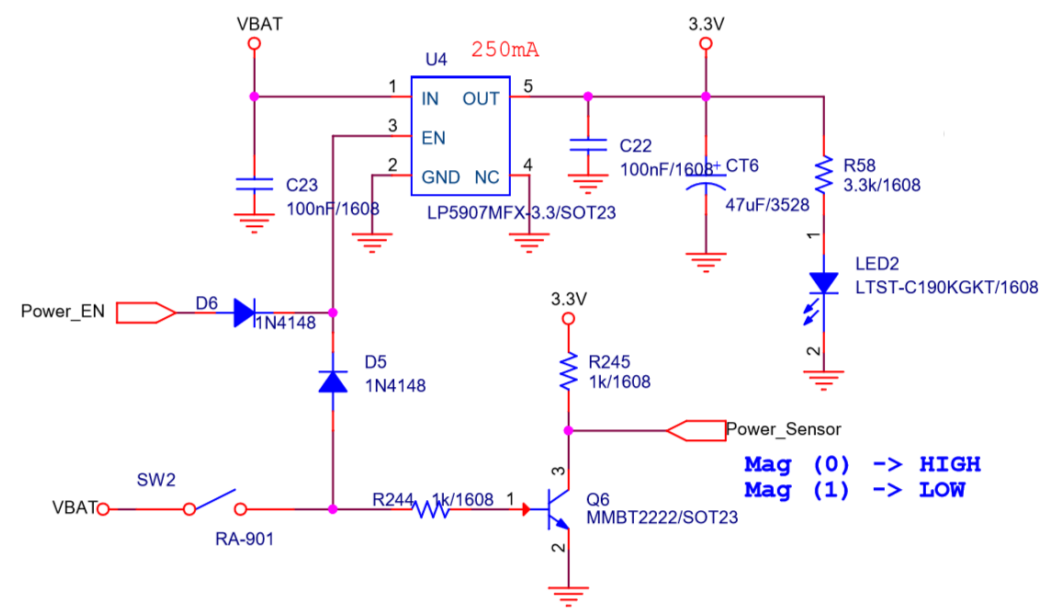

Figure 1. Power source circuit

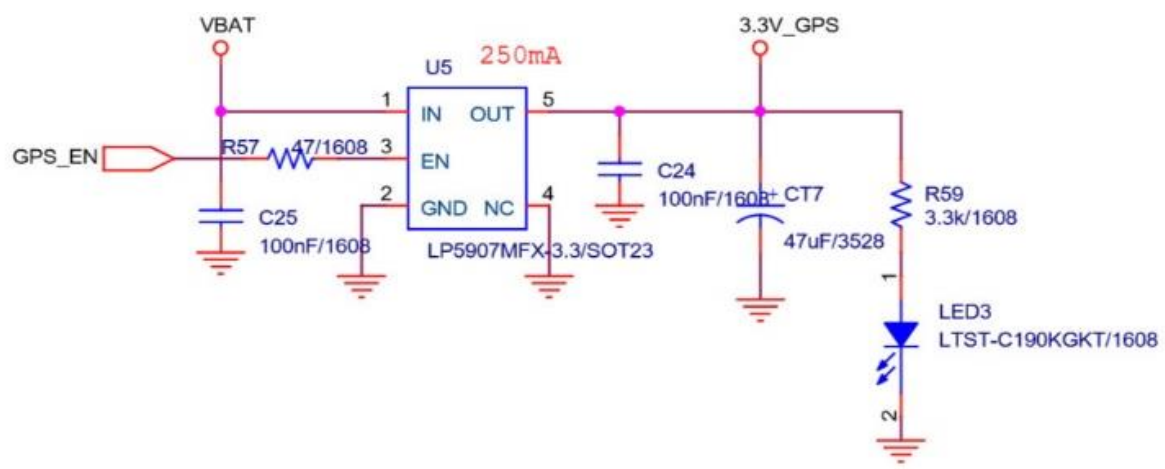

Figure 2. GPS control circuit by MCU

The MCU uses STM32L152RE from the STM company, and the GPS module uses MAX-M8Q from the u-blox company to reduce the position error using A-GPS. The LPWA module was made by Samtech's SX1276 RF module. We have developed software to drive this module. Figure 3 is a block diagram of the software functions in this module. The interface was designed using interlocking equipment and an internal module, and the execution concept was designed by unit software [22, 23]. 


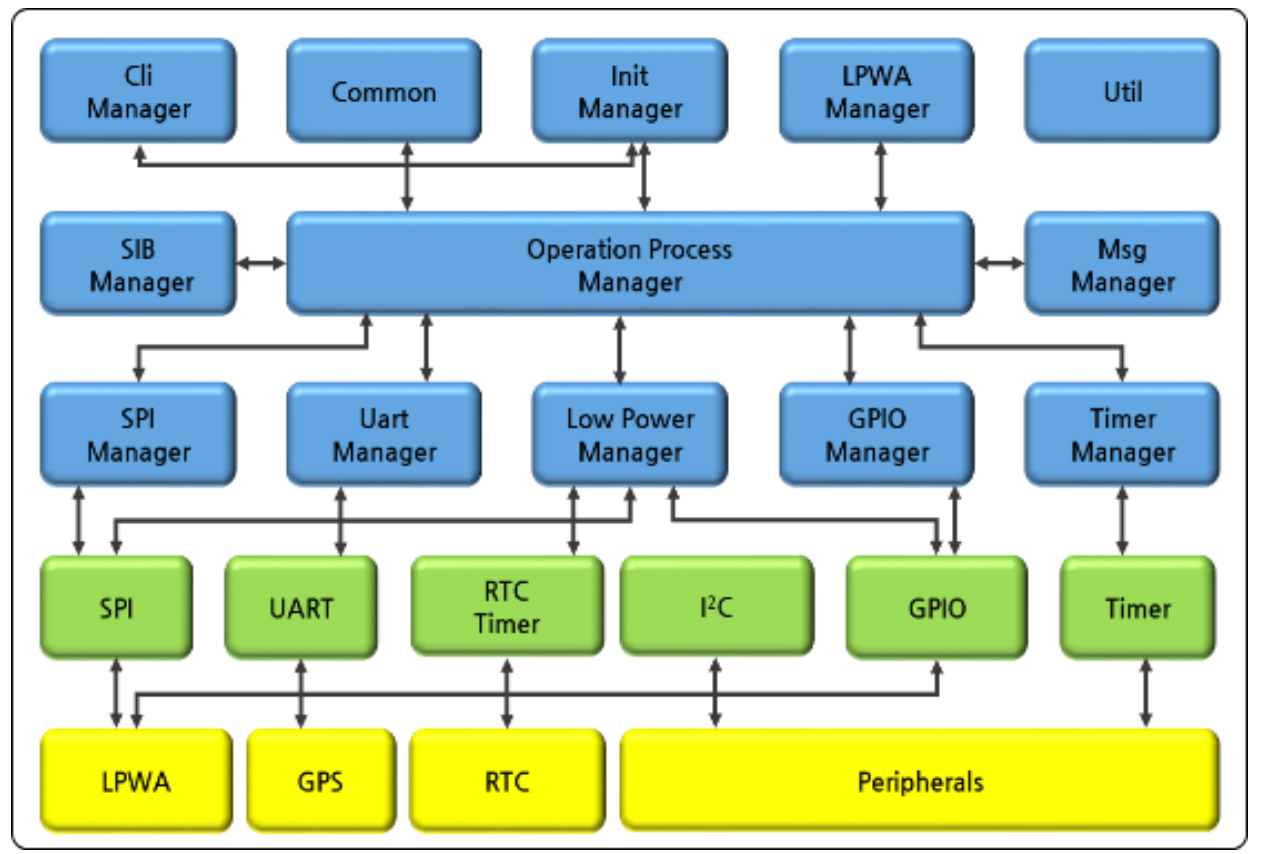

Figure 3. Software block diagram for maritime IoT module based on LPWA

\section{METHOD FOR MARITIME OBSERVATION}

The marine IoT module was used to develop marine control systems. Figure 4 shows the configuration of the ocean observation system proposed in this paper. The marine observation system receives data from a number of marine IoT modules. In order to receive data from a plurality of marine IoT modules, communication is performed by dividing them into a registration packet and a request packet. Figure 5 shows the communication procedure. The data collected by the marine IoT module is GPS data. GPS data are received, and latitude and longitude are transmitted to the ocean observation system. The ocean has a high tide and a low tide, so the position of the marine IoT module fixed on the floor changes incrementally, and the reception intensity of the signal generated in the communication changes, facilitating observation of the ocean. In this study, only information of GPS is received by the marine IoT module; however, various modules required for ocean observation can be attached, and information can be requested.

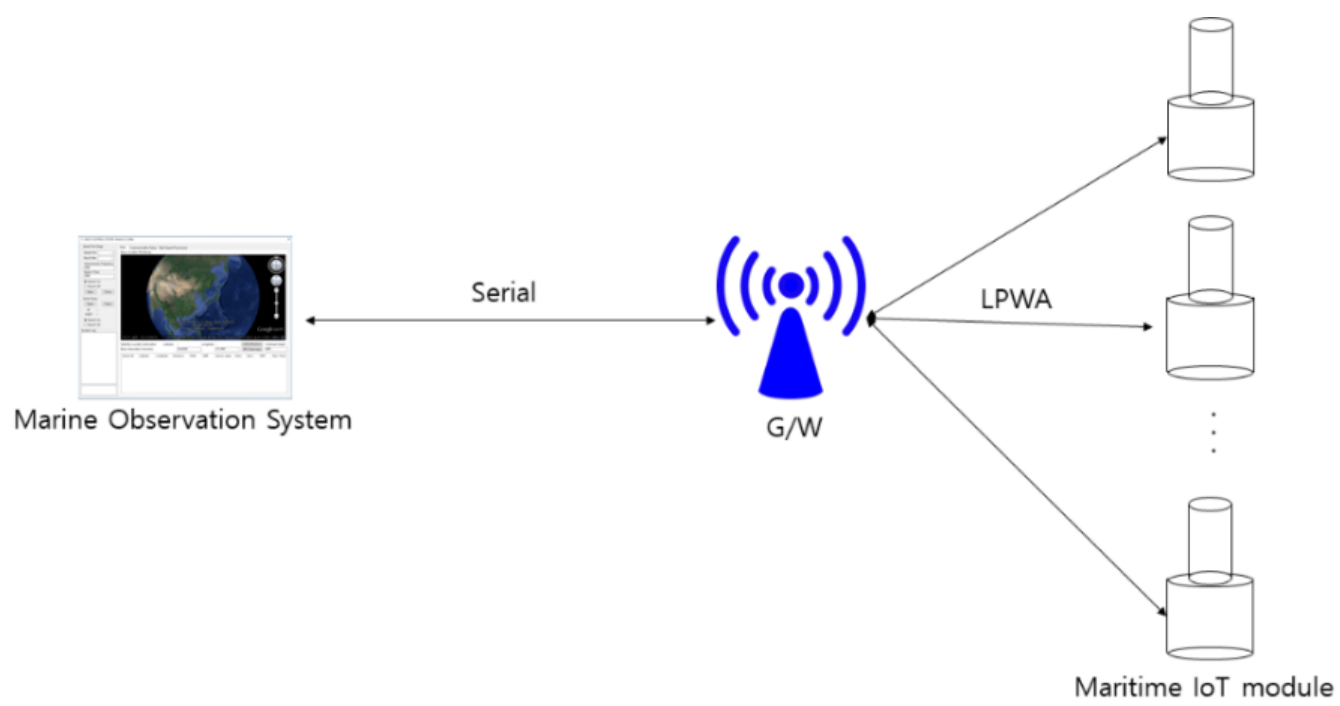

Figure 4. Configuration of ocean observation system 


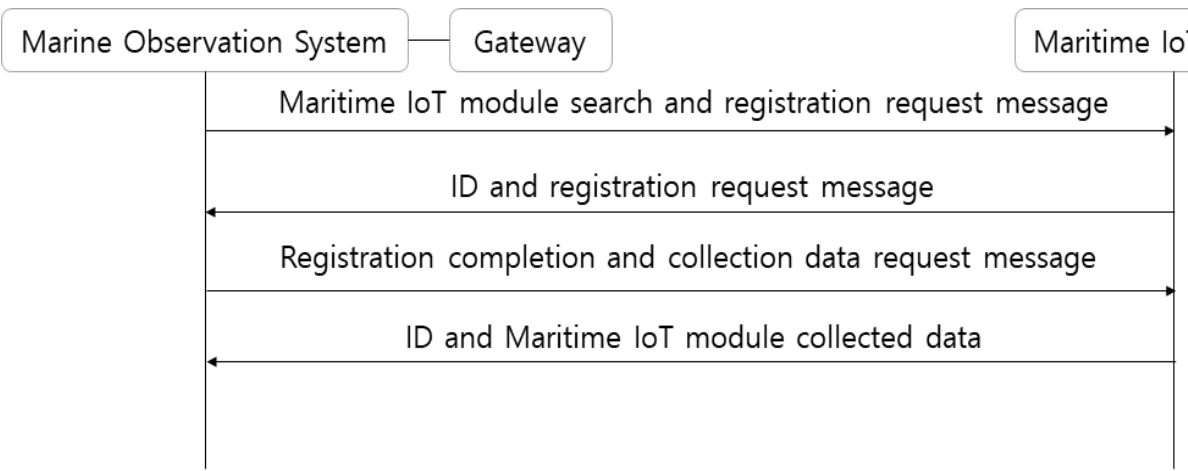

Figure 5. Communication procedure for data collection

\section{RESULT AND DISCUSSION}

The experiment was conducted with reference to data from publications provided by Lineliumd, a European LoRaWAN hardware provider, and papers related to performance when LoRaWAN was applied in a city center $[15,16,24,25]$. The experimental environment was constructed to evaluate the performance of the proposed marine IoT module based marine observation system using LPWA communication. The experimental environment is composed of an LoS state. The gateway installed in the experimental environment was installed about $10 \mathrm{~m}$ from the ground. The packet was transmitted under the control of the marine observation system. In order to check the message loss, the number of transmissions and the number of receivers were measured. The transmission rate was improved through one Ack message. The main parameters of LPWA used in the experiment are shown in Table 1.

Table 1. Maritime IoT module parameter values

\begin{tabular}{cc}
\hline Parameter & Value \\
\hline Frequency & $918.3 \mathrm{MHz}$ \\
Spreading Factor & 12 \\
Bandwidth & $125 \mathrm{kHz}$ \\
Coding Rate & $4 / 5$ \\
Transmit Power (uplink/downlink) & $14 \mathrm{dBm} / 20 \mathrm{dBm}$ \\
Antenna Gain & $2 \mathrm{dBi}$ \\
\hline
\end{tabular}

The experiment was carried out in Jangheung City, South Jeolla Province, Korea. Figure 6 shows the configured experimental environment. The gateway is installed on the second floor of the building. To perform serial communication, the marine observation system is installed next to the gateway. The marine IoT module is installed in a special case. Marine IoT modules are deployed in the sea using ships.

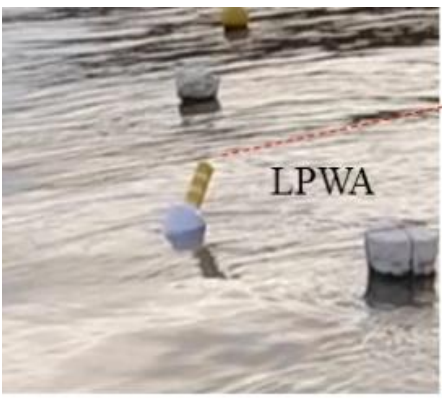

Maritime IOT module

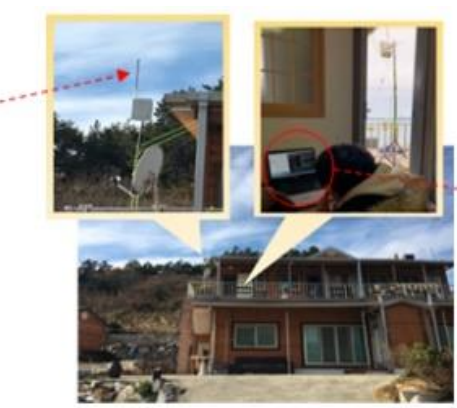

Installing the Gateway

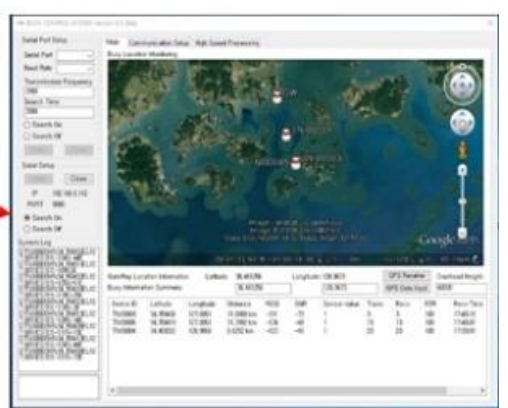

Marine Observation System

Figure 6. Actual experiment environment 
After establishing the experimental environment, two experiments were conducted. The first experiment measures the communication signal strength over distance, and confirms whether communication is possible up to $10 \mathrm{~km}$. The second experiment is to confirm that the marine IoT module placed on the sea receives the time-varying data, and it is identical to the ocean change. Three marine IoT modules were used for the distance test. The three modules have the IDs TNI00003, TNI00004, and TNI00005. The TNI00004 and TNI00005 modules were used for communication signal strength experiments. This test was performed at distances of $5 \mathrm{~km}$ and $10 \mathrm{~km}$ from the gateway. The reception results measured at each distance are shown in Figure 7 and 8.

The actual position of the marine IoT module was tracked using the GPS distance calculation formula for the location data of the gateway and the GPS data. To calculate distance using GPS information, we need to calculate the horizontal distance and the vertical distance by using the hardness and latitude. The hypotenuse is then found using Pythagoras' theorem. As shown in (1) to (4) are formulas for the distance between two points. As a result of measuring the distance, it was confirmed that the marine IoT module TNI00004 placed at $5 \mathrm{~km}$ was actually located at a distance of $6.3 \mathrm{~km}$, and the marine IoT module TNI00005 placed at $10 \mathrm{~km}$ was located at a distance of $11.8 \mathrm{~km}$.

The signal reception strength of TNI00004 is -123 to $-113 \mathrm{dBm}$, at the minimum. The average is $118.345 \mathrm{dBm}$. The signal reception strength of TNI00005 is -125 to $-114 \mathrm{dBm}$, at the minimum. The average is $-120.909 \mathrm{dBm}$. Since the average is about -121 at $11.8 \mathrm{~km}$, it is considered that communication is possible at far distances, considering the maximum reception sensitivity. The maximum reception sensitivity of LPWA varies depending on the setting, but up to $-136 \mathrm{dBm}$ is allowed.

The second experiment was carried out using the marine IoT module TNI00003. The marine IoT module installed at the $11.8 \mathrm{~km}$ site collected data for about one day. More data exist, but are expressed as averages over time, since there are limitations to the representation. Figures 9 and 10 show the intensity of the signal versus time and the elevation of the sea level.

It can be seen that the communication signal strength increases when the sea level is low. In addition, it can be seen that the communication signal strength decreases when the sea level becomes higher. This allows us to observe ocean changes using communication signals. Through the results of Experiment 1 and Experiment 2, we have proved that it is possible to analyze sea level changes at specific locations using the information of the proposed system.

$$
\begin{aligned}
& d_{l a}=G_{l a}-M_{l a} \\
& d_{l o}=G_{l o}-M_{l o} \\
& A=\sin ^{2}\left(\frac{d_{l a}}{2}\right)+\cos \left(M_{l a}\right) \times \cos \left(G_{l a}\right) \times \sin ^{2}\left(\frac{d_{l o}}{2}\right) \\
& D=(2 \times \arcsin (A)) \times \text { earth_radius }
\end{aligned}
$$

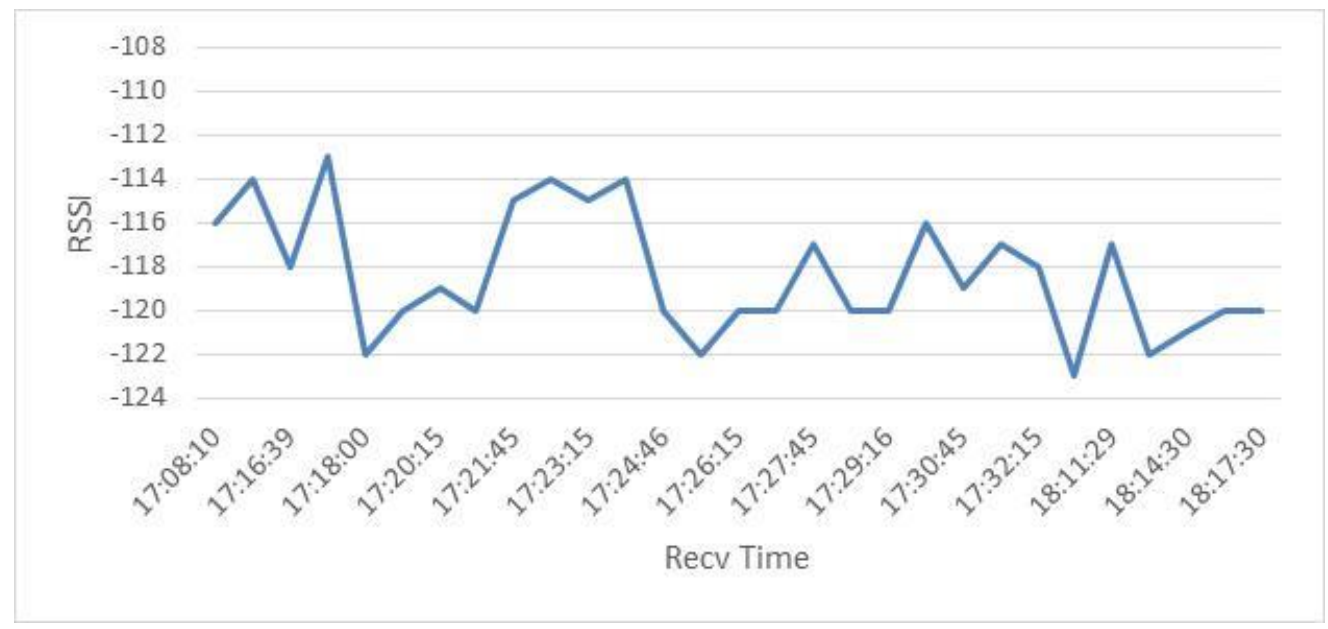

Figure 7. Test results at $5 \mathrm{~km}$ distance 


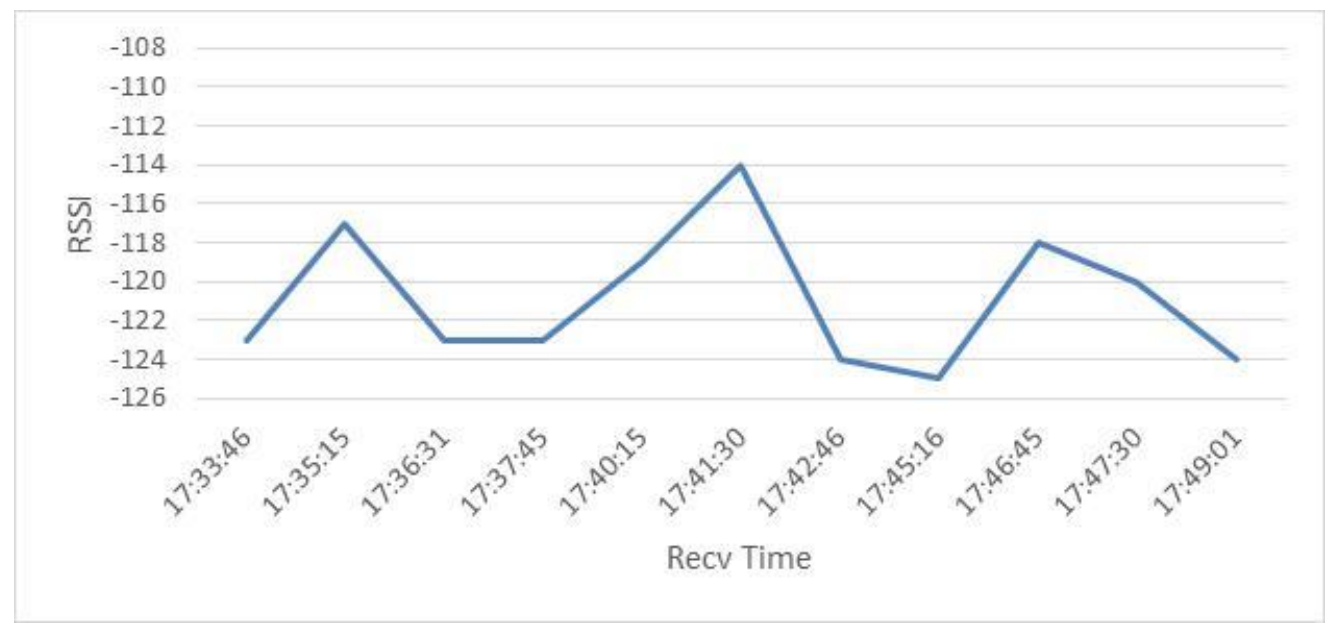

Figure 8. Test results at $10 \mathrm{~km}$ distance

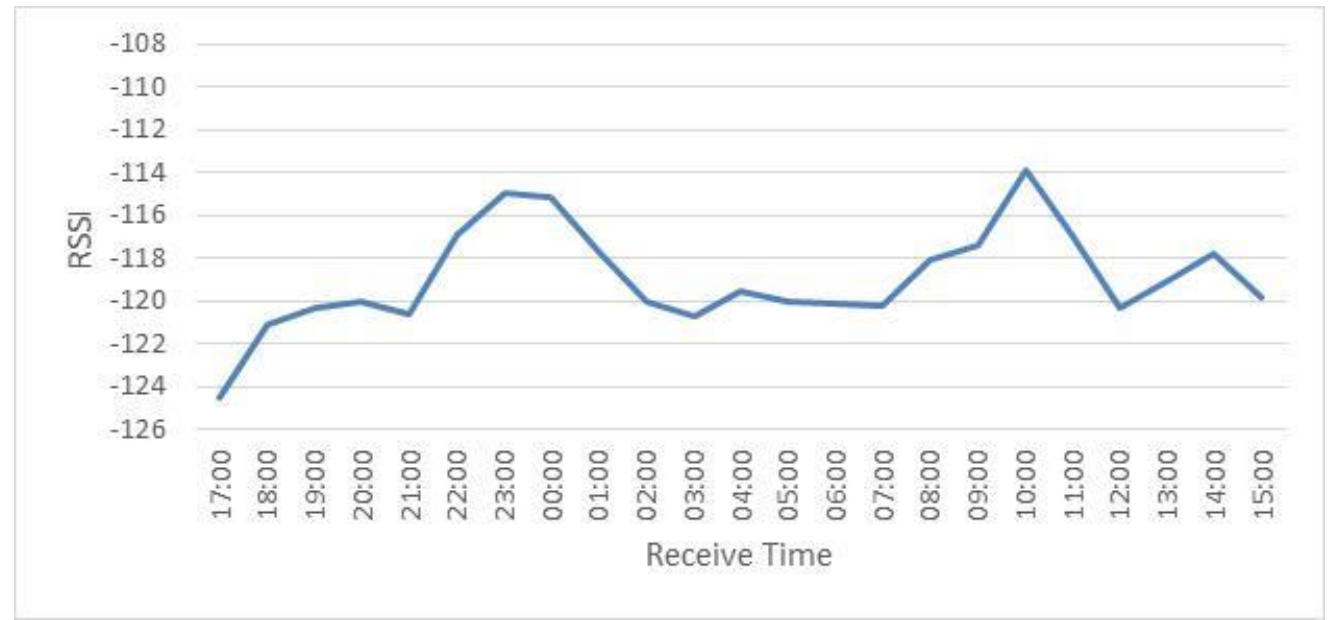

Figure 9. Communication signal intensity change over time

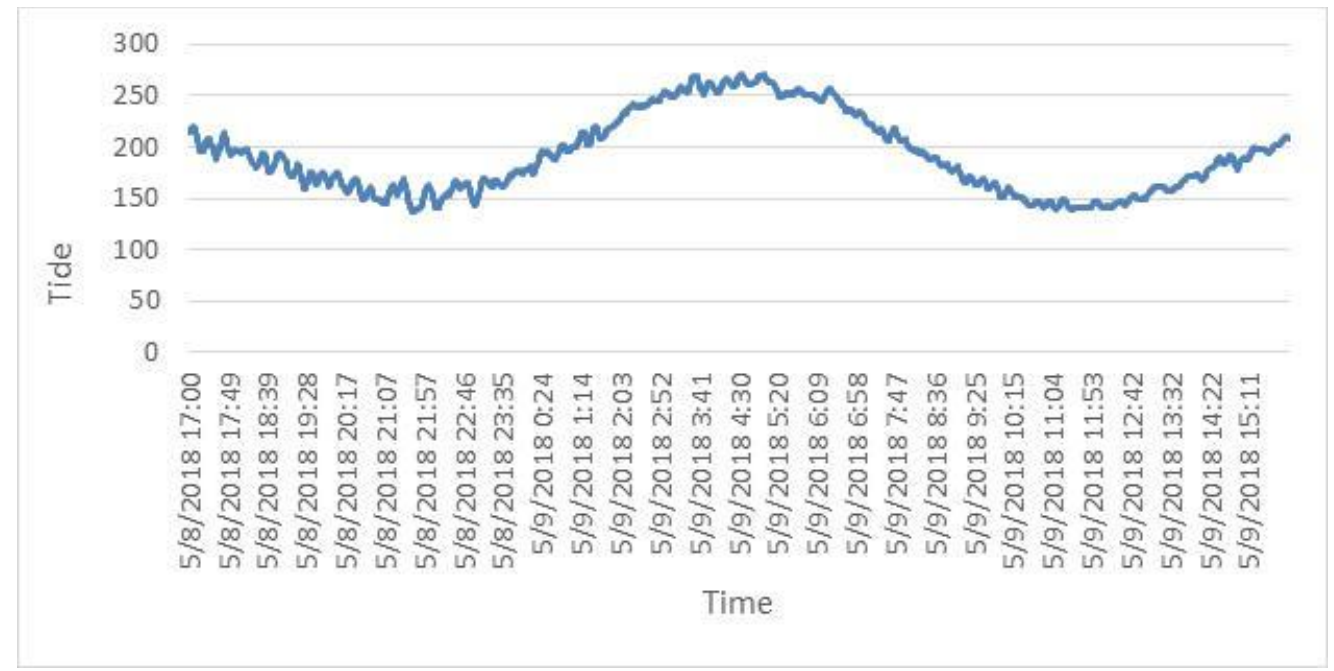

Figure 10. Sea level change over time 


\section{CONCLUSIONS}

We proposed a marine observation system based on a marine IoT module using LPWA communication. To validate the proposed system, we developed a marine IoT module, a gateway, and an ocean observation system that can receive information through LPWA. Using the developed system, we constructed the experimental environment in the sea and collected the signal strength of the location and communication of the marine IoT module. As a result, the distance between the gateway and the location of the marine IoT module was calculated using GPS. Further, it is proved that the intensity of the signal changes according to the change of the sea level when the intensity of the signal is compared with the change of the sea level. The system we have developed is part of a system that can provide marine IoT services. Therefore, we need to conduct further research to provide higher service. Further study should focus on attaching more sensors to the developed marine IoT module to gather more information. We will continue to experiment at longer distances.

\section{ACKNOWLEDGEMENTS}

This research was a part of the project titled "Development of Automatic Identification Monitoring System for Fishing Gears," funded by the Ministry of Oceans and Fisheries, Korea.

\section{REFERENCES}

[1] L Atzori, A. Lera, and G. Morabito, "The Internet of Things: A Survey," Computer Networks, vol. 54, no. 15, pp. 2787-2805, 2010.

[2] A. Whitmore, A. Agarwal, and L. Da Xu, "The Internet of Things-A Survey of Topics and Trends," Information Systems Frontiers, vol. 17, no. 2, pp. 261-274, 2015.

[3] S.S. Byun, "Measurement Allocation by Shapley Value in Wireless Sensor Networks," Journal of Information and Communication Convergence Engineering, vol. 16, no. 1, pp. 38-42, Mar 2018.

[4] H. Lim and H. Kim, "Low Power Time Synchronization for Wireless Sensor Networks Using Density-Driven Scheduling," Journal of Information and Communication Convergence Engineering, vol. 16, no. 2, pp. 84-92, 2018.

[5] Statista, IoT Connected Devices Installed Based Worldwide from 2015 to 2025 [Internet], Available: https://www.statista.com/statistics/471264/iot-number-of-connected-devices-worldwide/.

[6] J. Beh, K. Hur, W. I. Kim, and Y. I. Joo, "An Energy-Efficient MAC Protocol for Wireless Wearable Computer Systems," Journal of Information and Communication Convergence Engineering, vol. 11, no. 1, pp. 7-11, 2013.

[7] C. Perera, C. H. Liu, and S. Jayawardena, "The Emerging Internet of Things Marketplace from an Industrial Perspective: A survey," IEEE Transactions on Emerging Topics in Computing, vol. 3, no. 4, pp. 585-598, 2015.

[8] J. Ji and Y. Chung, "k-NN Join Based on LSH in Big Data Environment," Journal of Information and Communication Convergence Engineering, vol. 16, no. 2, pp. 99-105, 2018.

[9] K. Zheng, S. Zhao, X. Xiong, and X. Xiang, "Design and Implementation of LPWA-based Air Quality Monitoring System," IEEE Access, vol. 4, pp. 3238-3245, 2016.

[10] L. Da Xu, W. He, and S. Li, "Internet of Things in Industries: A Survey," IEEE Transactions on Industrial Informatics, vol. 10, no. 4, pp. 2233-2243, 2014.

[11] S. Husain, A. Prasad, A. Kunz, A. Papageorgiou, and J. Song, "Recent Trends in Standards Related to the Internet of Things and Machine-to-Machine Communications," Journal of Information and Communication Convergence Engineering, vol. 12, no. 4, pp. 228-236, 2014.

[12] R. S. Sinha, Y. Wei, and S. H. Hwang, "A Survey on LPWA Technology: LoRa and NB-IoT," ICT Express, vol. 3, no. 1, pp. 14-21, 2017.

[13] U. Raza, P. Kulkarni, and M. Sooriyabandara, "Low Power Wide Area Networks: An Overview," IEEE Communications Surveys \& Tutorials, vol. 19, no. 2, pp. 855-873, 2017.

[14] Y. S. Cho, J. Kwon, and S. Choi, "Design and Implementation of Wireless Sensor Network for Freeze Deyer," Journal of Information and Communication Convergence Engineering, vol. 13, no. 1, pp. 21-26, 2015.

[15] M. Taneja, "LTE-LPWA Networks for IoT Applications," International Conference on Information and Communication Technology Convergence (ICTC), pp. 396-399, 2016.

[16] U. Noreen, A. Bounceur, and L. Clavier, "A Study of LoRa Low Power and Wide Area Network Technology," Proceedings of the Advanced Technologies for Signal and Image Processing (ATSIP) 2017 International Conference, Fez, Morocco, October 22-24, 2017.

[17] G. A. Akpakwu, B. J. Silva, G. P. Hancke, and A. M. Abu-Mahfouz, "A Survey on 5G Networks for the Internet of Things: Communication Technologies and Challenges," IEEE Access, vol. 6, pp. 3619-3647, 2017.

[18] D. Ismail, M. Rahman, and A. Saifullah, "Low-Power Wide-Area Networks: Opportunities, Challenges, and Directions," Proceedings of the 19th International Conference on Distributed Computing and Networking (ICDCN), Varanasi, India, January 4-7, 2018.

[19] J. Petäjäjärvi, K. Mikhaylov, M. Hämäläinen, and J. Iinatti, "Evaluation of LoRa LPWAN Technology for Remote Health and Wellbeing Monitoring," Proceedings of the Medical Information and Communication Technology (ISMICT), 2016 10th International Symposium on IEEE, Worcester, MA, USA, pp. 20-23, 2016. 
[20] D. H. Lee and S. C. Kim, "Analysis of Computer Simulated and Field Experimental Results of LoRa Considering Path Loss under LoS and NLoS Environment," Journal of Korean Institute of Communications and Information Sciences, vol. 42, no. 2, pp. 444-452, 2017.

[21] A.S. Oh, "Development of a Smart Supply-Chain Management Solution Based on Logistics Standards Utilizing Artificial Intelligence and the Internet of Things," Journal of information and communication convergence engineering, vol. 17, no. 3, pp. 198-204, 2019.

[22] LoRa SX1276/77/78/79 Datasheet, Rev. 4. Semtech, 2015. Available online: http://www.semtech.com/ images/datasheet/sx1276_77_78_79.pdf (accessed on 8 September 2016).

[23] LoRa SX1272/73 Datasheet. Semtech, March 2015. Available online: http://www.semtech.com/images/ datasheet/sx1272.pdf (accessed on 8 September 2016).

[24] Libelium, Libelium Adds Extreme Range Wireless Connectivity to Waspmote IoT Sensors, Libelium World, 2014.

[25] A. Augustin, J. Yi, T. H. Clausen, and W. M. Townsley, "A Study of LoRa: Long Range \& Low Power Networks for the Internet of Things," sensor, vol. 16, no. 9, pp1-18, 2016.

\section{BIOGRAPHIES OF AUTHORS}
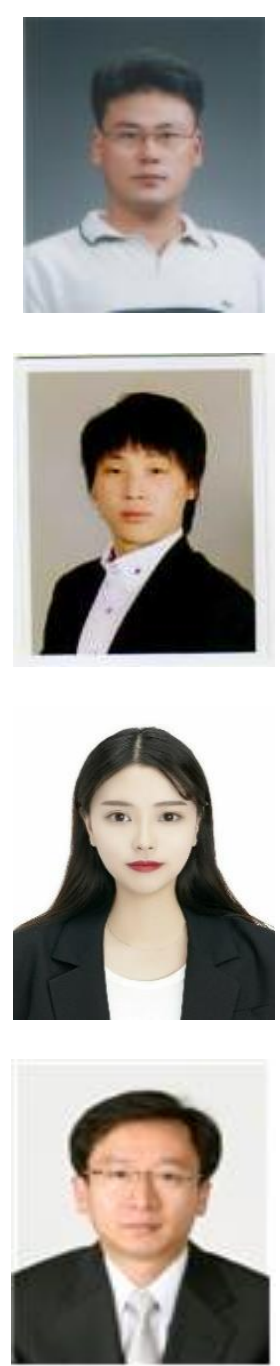

Se-Hoon Kim received his B.S degree from the Department of Electronics Engineering of the Hannam University, Daejeon, Korea, in 1993 and his M.S degree in Electronics Engineering from the Hannam University in 1995. Since January 1995, he has been serving as the Head of the R\&D Division at T\&Ie Co., Ltd., located in Seoul, Korea. His research interests include wireless/mobile communication, LPWA, and marine IoT and ICT Devices.

Min-Ho Jeon received his B.S degree from the Department of Game Digital Content of the Far East University, Umsung, Korea, in 2009 and his M.S. degree in Electrical, Electronics and Communication Engineering from the Korea University of Technology and Education (KOREATECH), Cheonan, Korea, in 2011. He is currently pursuing a Ph.D. Since June 2016, he has been with the Radio Communication Network (RCN) company, Daejeon, Korea, where he is currently a senior researcher. His research interests include wireless/mobile communication, wireless localization, and IoT.

Yeon-Ju Jo received her B.S degree in information communication engineering from Mokwon University, Daejeon, Korea, in 2018. Since June 2017, she has been with the Radio Communication Network ( $\mathrm{RCN})$ company, Daejeon, Korea, where she is currently a researcher. Her research interests include wireless/mobile communication, IoT, industrial IoT, and LPWA.

Chang-Heon Oh received his B.S. and M.S.E. degrees in telecommunication and information engineering from Korea Aerospace University, Kyunggi-Do, Korea, in 1988 and 1990, respectively. He received his $\mathrm{Ph} . \mathrm{D}$. degree in avionics engineering from Korea Aerospace University, Kyunggi-Do, Korea, in 1996. From February 1990 to August 1993, he was with Hanjin Electronics Co., where he was involved in the research and development of radio communication and monitoring systems. From October 1993 to February 1999, he was with the CDMA R\&D center of Samsung Electronics Co., where he was involved in the design and development of CDMA cellular systems and CDMA PCS systems for successful commercial CDMA deployment in Korea. Since March 1999, he has been with the School of Electrical, Electronics and Communication Eng., Korea University of Technology and Education (KOREATECH), Cheonan, Korea, where he is currently a professor. His research interests include wireless/mobile communication, wireless localization, IoT, and engineering education. 